\title{
Optical injection of charge current in quantum wires: Oscillations induced by excitonic effects
}

\author{
D. H. Marti, M.-A. Dupertuis, and B. Deveaud \\ Institute of Quantum Electronics and Photonics, Ecole Polytechnique Fédérale de Lausanne (EPFL), CH-1015 Lausanne, Switzerland
}

(Received 5 April 2004; revised manuscript received 19 January 2005; published 24 August 2005)

\begin{abstract}
We investigate the charge current that is optically injected by interference between one- and two-photon excitation in the presence of excitonic effects. We consider a realistic V-shaped quantum wire excited slightly below the band gap by two simultaneous femtosecond laser pulses of frequency $2 \omega$ and $\omega$ that interact, respectively, with the lowest $B_{1}$ and $A_{1}$ excitons. Using effective multiband Bloch equations for two-photon transitions, including the Coulomb interaction within the Hartree-Fock approximation, we show that, because of the different symmetry properties of the involved excitons, the generated charge current displays oscillations due to the quantum interference between the excitonic coherences.
\end{abstract}

DOI: 10.1103/PhysRevB.72.075357

\section{INTRODUCTION}

Coherent control of charge current generated by quantum interference between one- and two-photon absorption has attracted considerable interest, since the first measurements in semiconductors. ${ }^{1,2}$ In this context, various questions have been addressed, such as the relaxation due to electronphonon interaction, ${ }^{3}$ the effect of strain on the degree of spin polarization of the current, ${ }^{4}$ or the contribution of intersubband coherence on the THz emission. ${ }^{5}$ However, it remains an open question how such processes may be affected by the Coulomb interaction. Interesting phenomena may be expected, as it will be shown in this paper.

In the following, we apply the effective multiband Bloch equations introduced in Refs. 5 and 6 to the case of optical injection of current in the presence of excitonic effects. This first investigation concentrates on a one-dimensional system, a realistic V-shaped AlGaAs/As quantum wire (QWR). We give first results concerning the charge current generated in the QWR, and the associated terahertz emission, when the lowest $B_{1}$ and $A_{1}$ excitonic resonances are excited simultaneously by one- and two-photon transitions. Because of the different symmetry properties of the involved excitons, the injected charge current displays oscillations due to the quantum interference between the excitonic coherences. The oscillation frequency is given by the energy spacing between the excitons. This phenomenon has the same origin as the polarization interference between excitons with different energy, observed in quantum wells by four-wave mixing. ${ }^{7-9}$ However, the excitation scheme described in this study makes the resulting modulation of the interband coherence also appear as oscillations in the charge current, which is directly related to the occupation of the bands.

The text is organized as follows. In Sec. II we introduce the theory and detail of the excitation scheme that is considered. Then, in Sec. III we show and discuss the numerical results. The conclusions are drawn in Sec. IV.

\section{THEORY}

We consider a QWR simultaneously excited by two laser pulses polarized along the wire-i.e., along the symmetry
PACS number(s): 78.67.-n, 78.30.Fs, 78.20.Bh axis $x$ (Fig. 1). The two fields of frequency $\omega_{1}=2 \omega$ close to the band gap and $\omega_{2}=\omega$ close to the half of the band gap induce, respectively, quasiresonant one- and two-photon interband transitions. It must be pointed out that the excitation scheme is slightly different from the one usually used for coherent control of current injection, ${ }^{1-5}$ where a large number of carriers are injected through resonant valence-toconduction band transitions. Here we choose optical frequencies $\omega_{1}$ and $2 \omega_{2}$ slightly below the band gap energy in order to build up essentially the lowest excitonic coherences. Let us first describe the QWR we consider (Sec. II A). We shall then summarize the involved excitonic selection rules (Sec. II B) and introduce the equations of motion of the system (Sec. II C). Further details about the excitation scheme are finally given in Sec. II D.

\section{A. V-shaped quantum wire}

The QWR is a V-shaped $\mathrm{Al}_{x} \mathrm{Ga}_{1-x} \mathrm{As} / \mathrm{GaAs}$ QWR $(x$ $=0.337$ ) orientated with respect to the crystal main symmetry axes, as shown in the inset of Fig. 1. Since the inversion asymmetry in GaAs has only very small effects on the valence band structure, it is neglected here. Within this approximation, the QWR point group is $\mathbf{C}_{2 v}$ with the two symmetry planes $\sigma_{x}$ and $\sigma_{y}$ defined by their normal axes $x$ $([1 \overline{1} 0])$ and $y([110])$, respectively, and the little group of $k$ is $C_{s}$ (classification adopted in Ref. 10).

For the band-structure computations, the exact contour is extracted from a transmission electron microscope (TEM) picture (inset in Fig. 1). The confined electronic states in the GaAs/AlGaAs QWR and the related momentum matrix elements were calculated as in Refs. 10 and 11 with $\mathbf{k} \cdot \mathbf{p}$ theory, the bulk conduction band is a doubly degenerate S-like band, and the bulk valence band is described by the four-band Luttinger Hamiltonian. The bulk momentum matrix elements between a conduction band and a valence band are given by the two-by-four Kane matrices, ${ }^{12}$ whereas the elements between two conduction bands or two valence bands are equal to zero due to symmetry. The bulk band-structure parameters are shown in Table I, and the energy subbands of the QWR are displayed in Fig. 1. The bands are labelled as follows: $c_{i}$ denotes the $i$ th pair of conduction subbands, starting from the 


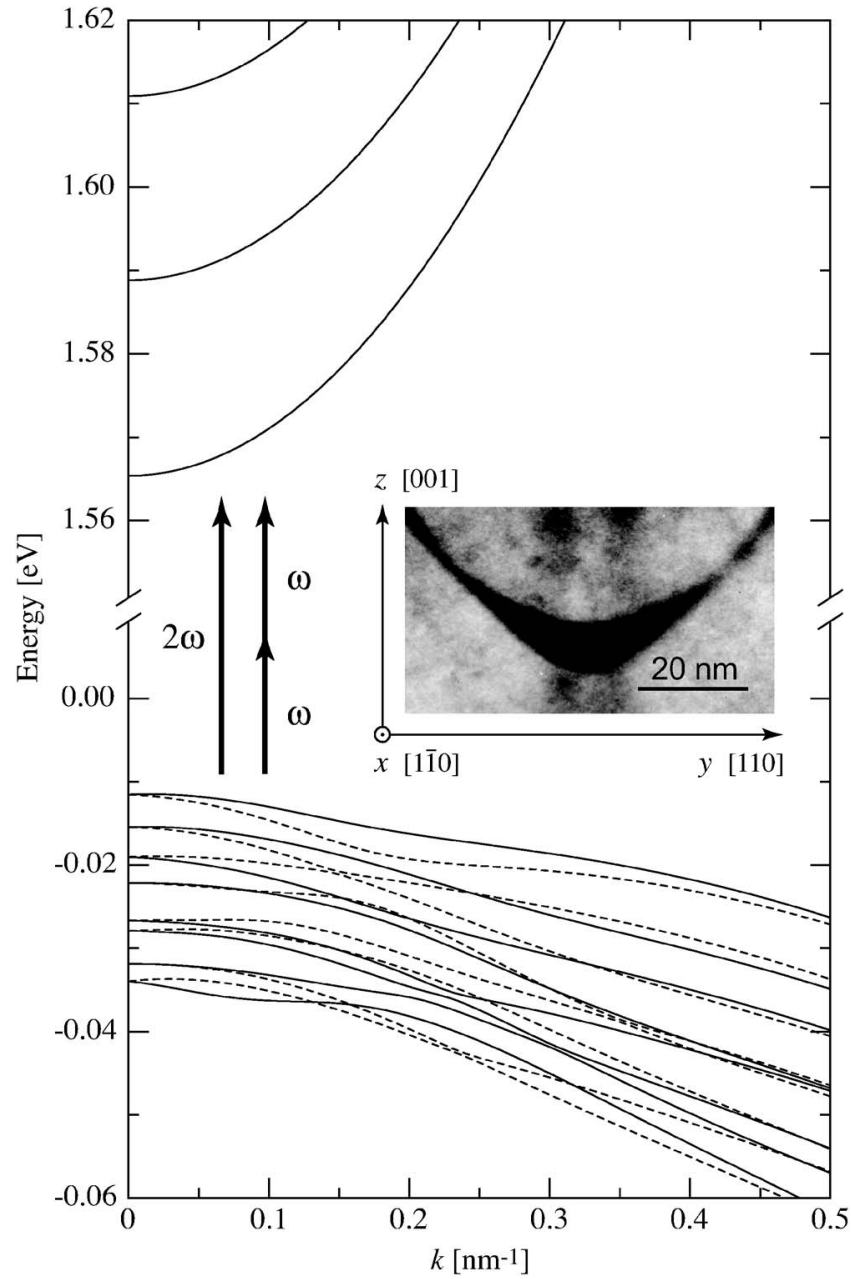

FIG. 1. Band structure of a V-shaped AlGaAs/GaAs quantum wire. Two bands that are degenerate at zone center are mutually conjugate by time reversal and belong to the irreps ${ }^{1} E_{1 / 2}$ (solid line) and ${ }^{2} E_{1 / 2}$ (dashed line) of the group $\mathbf{C}_{s}$ (the little group of $k$ ). The QWR is excited by two optical pulses of frequency $\omega$ and $2 \omega$ with $2 \omega$ slightly below the band gap. The inset shows a TEM picture of the quantum wire considered in this study.

bottom, and $v_{i}$ denotes the $i$ th pair of valence subbands, starting from the top.

\section{B. Excitonic selection rules}

Excitonic quantum numbers may be defined from the irreducible representations (irreps) of product states of single particles, since the Coulomb interaction always belongs to the invariant representation and thus mixes only product states with a given representation. Insofar as the wave vector of the electromagnetic field is neglected, only the excitons with center-of-mass wave vector equal to zero are optically active. As a consequence, all optically active excitons exhibit the zone center symmetry and can be labelled by the irreps of the point group of the QWR. ${ }^{10}$ Furthermore, excitons being product states of two particles with half-integer spin, their total spin is an integer. By contrast to the single particle case, excitons therefore belong to the irreps of the single group. ${ }^{10}$
In QWRs with $\mathbf{C}_{2 v}$ symmetry, the optically active excitons can consequently be classified according to the four onedimensional irreps denoted by $A_{1}, B_{1}, A_{2}$, and $B_{2}$ (notations of Ref. 15).

Let $|n, k\rangle$ be the electronic state of wave vector $k$ in the subband $n$. In the dipole approximation, the optical coupling between a state in the conduction subband $c$ and a state in the valence subband $v$ is described by the linear interaction term $\pi_{c v, k}^{x}$ for resonant one-photon transitions and by the effective quadratic interaction term $\Sigma_{n} \pi_{c n, k}^{x} \pi_{n v, k}^{x} /\left(\Omega_{n v, k}-\omega\right)$ for resonant two-photon transitions. ${ }^{5,6}$ The matrix elements of the momentum operator $\pi^{x}$ including spin-orbit coupling are defined by $\pi_{m n, k}^{x}=\left\langle m, k\left|\pi^{x}\right| n, k\right\rangle$ and $\Omega_{m n, k}$ denotes the interband frequency between the bands $m$ and $n$ at wave vector $k$. Under the spatial symmetry operations, the linear operator $\pi^{x}$ transforms like the Cartesian component $x$, whereas the quadratic operator $\Sigma_{n} \pi^{x}|n, k\rangle\langle n, k| \pi^{x} /\left(\Omega_{n v, k}-\omega\right)$ transforms like the product of two Cartesian components $x x$. Taking into account these symmetries, the excitonic selection rules can be derived. We conclude that for one-photon excitation the light polarized along the QWR interacts with the class of excitons $B_{1}$, whereas by two-photon excitation with light polarized along the symmetry axis $x$, only excitons of the type $A_{1}$ are accessed.

\section{Equations of motion and charge current}

The dynamics is described by the effective multiband Bloch equations introduced in Refs. 5 and 6, which include nonlinear processes such as two-photon transitions or AC Stark shifts. They are extended here to account for the Coulomb interaction within the Hartree-Fock approximation. Indeed, we need an equation of motion for the reduced density matrix, including both the quasiresonant effective twophoton transitions and the Coulomb repulsion. However, we neglect the cross terms between the electromagnetic field and the Coulomb interaction that result from the perturbative expansion. Each interaction is treated separately and the resulting effective interactions are eventually added. In the following, we thus give only the equation of motion, and the involved approximations, in the absence of electromagnetic field. When expressed in the same rotating frame, the Coulomb interaction terms can then simply be added to the effective multiband Bloch equations [Eq. (23) in Ref. 5, see also Ref. 6].

First, we disregard the Hartree (or direct) term. Note that it cancels exactly ${ }^{16}$ if one neglects the Coulomb-induced transitions between different bands and the wave function dependence of the Coulomb matrix element. Second, we neglect the transitions between conduction and valence bands induced by Coulomb scattering. Finally, as we restrict ourselves to the low density regime, we also neglect the Coulomb coupling between electrons in the conduction subbands or between electrons in the valence subbands. To summarize, keeping only the Fock (or exchange) term, we are left with the equation of motion, 
TABLE I. Bulk band-structure parameters (Ref. 14) of $\mathrm{Al}_{x} \mathrm{Ga}_{1-x}$ As used in this study.

\begin{tabular}{lccc}
\hline \hline Symbol & Parameter & $\mathrm{Al}_{x} \mathrm{Ga}_{1-x} \mathrm{As}$ & Unit \\
\hline$m_{c}$ & $\begin{array}{c}\text { Effective mass of the } \\
\text { conduction band }\end{array}$ & $0.0665+0.0835 x$ & $\left(m_{0}\right)$ \\
& Luttinger parameter & $6.790-3.000 x$ & $(-)$ \\
$\gamma_{1}$ & Luttinger parameter & $1.924-0.694 x$ & $(-)$ \\
$\gamma_{2}$ & Luttinger parameter & $2.681-1.286 x$ & $(-)$ \\
$\gamma_{3}$ & Kane parameter & 28.8 & $(\mathrm{eV})$ \\
$E_{P}$ & Energy gap & $1.519+1.247 x$ & $(\mathrm{eV})$ \\
$E_{g}$ & Band offsets & $68 / 32$ & $(-)$ \\
$\Delta E_{c} / \Delta E_{v}$ & (e)
\end{tabular}

${ }^{\mathrm{a}}$ For future calculations we suggest to use the better formula $E_{g}(x)=1.5194+1.36 x+0.22 x^{2}$ (Ref. 13).

$$
\begin{aligned}
\partial_{t} \rho_{m m^{\prime}, k}= & -i \Omega_{m m^{\prime}, k} \rho_{m m^{\prime}, k} \\
& +\frac{i}{\hbar} \sum_{n n^{\prime} n^{\prime \prime}} \sum_{q}\left[V_{q}\right]_{k, k-q ; k-q, k}^{m, n^{\prime \prime} ; n^{\prime}, n} \rho_{n^{\prime \prime} n^{\prime}, k-q} \rho_{n m^{\prime}, k} \\
& -\frac{i}{\hbar} \sum_{n n^{\prime} n^{\prime \prime}} \sum_{q} \rho_{m n, k} \rho_{n^{\prime} n^{\prime \prime}, k-q}\left[V_{q}\right]_{k, k-q ; k-q, k}^{n, n^{\prime} ; n^{\prime \prime}, m^{\prime}},
\end{aligned}
$$

restricted to the Coulomb matrix elements $\left[V_{q}\right]_{\ldots}^{c, c^{\prime} ; v, v^{\prime}}$ and $\left[V_{q}\right]_{\ldots}^{v, v^{\prime} ; c, c^{\prime}}$ (the band indices $c$ and $v$ denote conduction and valence bands, respectively). The notations are identical to the one used in Refs. 5 and $6, \rho_{m m^{\prime}, k}$ and $\Omega_{m m^{\prime}, k}$ are, respectively, the reduced density matrix element and the bare (nonrenormalized) interband frequency between the electronic states of band $m$ and $m^{\prime}$ with wave vector $k$. Band-gap renormalization is included in the remaining terms in Eq. (1), and the exchange energy is taken into account. However we neglect screening and the so-called "Coulomb-hole" correction, which restricts the validity to a relatively low-density regime. The Coulomb matrix elements are given by

$$
\begin{aligned}
{\left[V_{q}\right]_{k-q, k ; k^{\prime}+q, k^{\prime}}^{m, n ; m^{\prime}, n^{\prime}}=} & \frac{e^{2}}{\epsilon_{0} L} \int \psi_{k-q}^{m}(\mathbf{r})^{\dagger} \psi_{k}^{n}(\mathbf{r}) e^{i q\left(x-x^{\prime}\right)} G_{q}\left(\mathbf{r}_{\perp}, \mathbf{r}_{\perp}^{\prime}\right) \\
& \times \psi_{k^{\prime}+q}^{m^{\prime}}\left(\mathbf{r}^{\prime}\right)^{\dagger} \psi_{k^{\prime}}^{n^{\prime}}\left(\mathbf{r}^{\prime}\right) d^{3} \mathbf{r} d^{3} \mathbf{r}^{\prime}
\end{aligned}
$$

where the $\psi_{k}^{n}(\mathbf{r})$ 's are electronic wave functions and is the real Green's function of the equation

$$
\left\{\boldsymbol{\nabla}\left[\epsilon\left(\mathbf{r}_{\perp}\right) \boldsymbol{\nabla}\right]-q^{2} \epsilon\left(\mathbf{r}_{\perp}\right)\right\} G_{q}\left(\mathbf{r}_{\perp}, \mathbf{r}_{\perp}^{\prime}\right)=\delta\left(\mathbf{r}_{\perp}-\mathbf{r}_{\perp}^{\prime}\right),
$$

which is the Fourier transform (in the unconfined direction) of the 3D Poisson equation. $L$ is the length of the QWR and $\mathbf{r}_{\perp}=(y, z)$ designates the projection of the position $\mathbf{r}$ $=(x, y, z)$ on the plane perpendicular to the QWR. The position dependent dielectric function $\epsilon\left(\mathbf{r}_{\perp}\right)$ accounts for the material variation in the heterostructure. In the bulk case, $\epsilon$ is constant and the solution of Eq. (3) reduces to the wellknown function $G_{q}=-1 /\left(\epsilon q^{2}\right)$.

It is reasonable to assume that the Coulomb contribution of filled bands is already taken into account by the effective bulk parameters (band energies, effective masses, etc.). The occupation numbers $\rho_{v v, k}$ of the electrons in the valence bands arethus replaced by $\rho_{v v, k}-1$ in the Coulomb terms.
The resulting Coulomb interaction terms (restricted to the bands described by the reduced density matrix) can now be added to the effective multiband Bloch equations, insofar they are expressed in the same rotating frame. The required Coulomb matrix elements were calculated following Ref. 17 by solving the Poisson equation with position dependent dielectric function.

The effective equations of motion are solved for a reduced density matrix describing the three lowest pairs of conduction bands and the four highest pairs of valence bands (Fig. 1). The two bands defining a pair are conjugate by timereversal symmetry. In order to correctly describe the twophoton transitions, one should in principle take into account a complete set of intermediate states [the sum over $n$ in Eqs. (25) and (26) of Ref. 5, see also Ref. 6]. In practice however, we restrict ourselves to the 12 lowest pairs of conduction bands and the 24 highest pairs of valence bands.

The optically generated charge current is given by the "low frequency" part of the polarization current [Eq. (31) of Ref. 5, see also Ref. 6],

$$
\mathbf{j}=\frac{1}{L} \sum_{k}\left(\sum_{c c^{\prime}} \boldsymbol{\Pi}_{c c^{\prime}, k} \rho_{c^{\prime} c, k}+\sum_{v v^{\prime}} \boldsymbol{\Pi}_{v v^{\prime}, k} \rho_{v^{\prime} v, k}\right) .
$$

Note that the wave vector $k$ is here one dimensional and the polarization current is normalized by the length $L$ of the QWR.

The decoherence and the thermalization of the carrier distributions are described by a unique phenomenological relaxation time $\tau$ [Eqs. (27) and (28) in Ref. 5]. This is a simple and convenient way to model complex microscopic relaxation mechanisms such as electron-phonon and electronelectron scattering. A detailed description of these processes in the case of current injection would be of great interest. This issue certainly deserves further investigations, but it is also a challenging task from the computational point of view.

\section{Excitation scheme}

The two simultaneous laser pulses are described by the vector potentials $\mathbf{A}_{1} \cos (2 \omega t+\phi)$ and $\mathbf{A}_{2} \cos (\omega t)$. With respect to the notations used in Ref. 5, the frequencies and phases of these fields correspond to $\phi_{1}=\phi, \phi_{2}=0$, and $\omega_{1}$ $=2 \omega_{2}=2 \omega$. The two pulses have a duration of $100 \mathrm{fs}$ at half- 


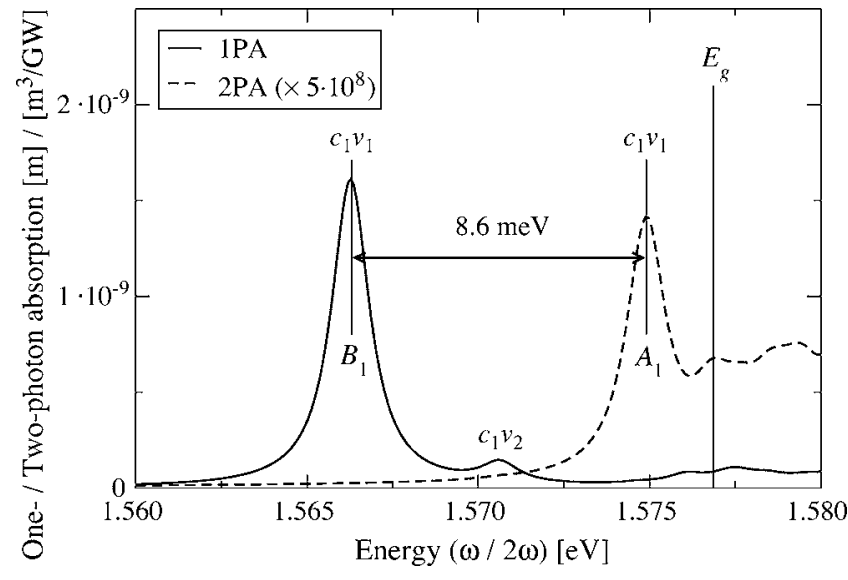

FIG. 2. Lowest $B_{1}$ and $A_{1}$ excitonic resonances in the onephoton absorption (1PA) and two-photon absorption (2PA), respectively. The absorption spectra are calculated for thermalized carrier distributions that correspond approximatively to the carrier densities excited in the configuration for current injection used in this paper. Decoherence time, $\tau=1 \mathrm{ps} . E_{g}$, energy gap of the QWR.

maximum (of intensity) and are copolarized along the QWR ( $x$ axis). The optical frequency $2 \hbar \omega=1.57 \mathrm{eV}$ corresponds to an excitation energy in the middle between the two lowest excitonic resonances for light polarized along the $x$ axis, namely the lowest $B_{1}$ exciton in the one-photon absorption spectrum and the lowest $A_{1}$ exciton in the two-photon absorption spectrum (Fig. 2). The absorption spectra are calculated by solving the same effective multiband Bloch equations, when the QWR is excited only by the $\mathbf{A}_{1}$ or $\mathbf{A}_{2}$ pulse, they are extracted from the Fourier transform of the highfrequency part of the polarization current. The dominant contribution to both excitonic resonances is given by the lowest conduction band and the highest valence band denoted by $c_{1}$ and $v_{1}$, respectively. The spectral widths of the pulses are sufficiently large to cover both resonances. The peak intensities are chosen to get a high contrast in the interference, $7 \mathrm{MW} / \mathrm{cm}^{2}$ and $10 \mathrm{GW} / \mathrm{cm}^{2}$ for the $\mathbf{A}_{1}$ and $\mathbf{A}_{2}$ pulse, respectively. The calculations are essentially done for a long relaxation time $\tau=1 \mathrm{ps}$. A detailed discussion of complete one- and two-photon absorption spectra in QWRs is given in Ref. 18.

\section{RESULTS AND DISCUSSION}

\section{A. Oscillating charge current}

In Fig. 3, we show the charge current (4)-i.e., the lowfrequency part of the polarization current-injected along the QWR for two values of the phase difference between the pulses, $\phi=0$ and $\phi=\pi / 2$. The results are displayed for a long relaxation time ( $\tau=1 \mathrm{ps})$, and compared to the values obtained in the same configuration but without Coulomb interaction. The power spectrum $P(\nu) \propto\left|\int_{-\infty}^{+\infty}\left(\partial_{t} \mathbf{j}\right) e^{i 2 \pi \nu t} d t\right|^{2}$ of the associated terahertz emission is displayed in Fig. 4.

When the Coulomb interaction is taken into account within the Hartree-Fock approximation, a strongly oscillating charge current appears. First, we note that the oscillations disappear, when the Coulomb interaction is neglected. This

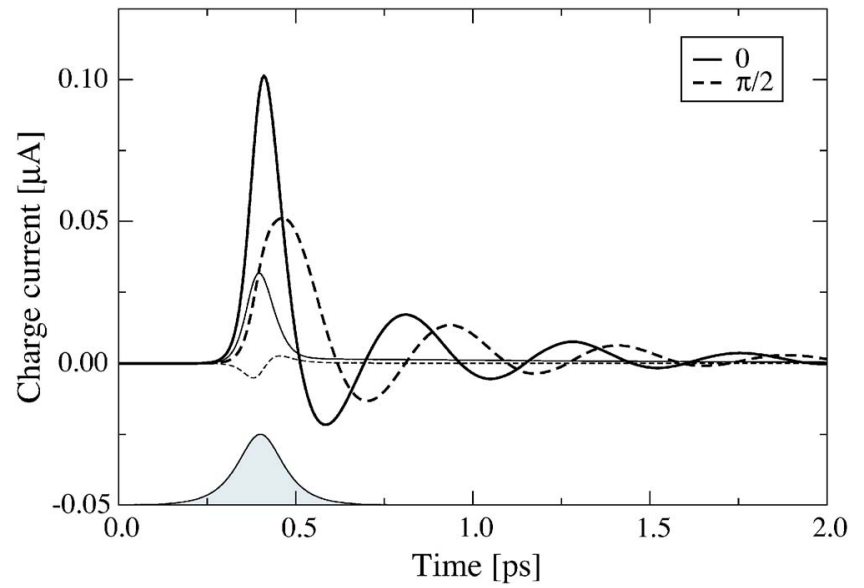

FIG. 3. (Color online) Charge current along the V-shaped quantum wire for two different optical phases, $\phi=0$ (solid line) and $\phi$ $=\pi / 2$ (dashed line). Thick lines, the Coulomb interaction is taken into account within the Hartree-Fock approximation. Thin lines, the Coulomb interaction is neglected. Relaxation time $\tau=1 \mathrm{ps}$. The shaded curve on the bottom of the figure shows the time dependence of the optical pulses in the QWR.

clearly indicates that excitonic resonances are involved. Second, the oscillations subsist even after the optical pulses vanished. We also checked that the oscillation frequency does not depend on the pulse frequencies, insofar as the same resonances are excited. Note that the current is enhanced by the Coulomb interaction, because the optical pulses (with frequency below the band gap) mainly excite excitonic resonances. Finally, as expected there is no current injected when only one beam is present.

\section{B. Charge current injection: Two contributions}

The oscillating current is present in both phase configurations $(\phi=0$ and $\phi=\pi / 2)$. However, as it can be seen from the terahertz spectra, an additional (broader) contribution appears for $\phi=0$.

To investigate this phase dependence, let us first remind the phenomenon of current injection by interference between

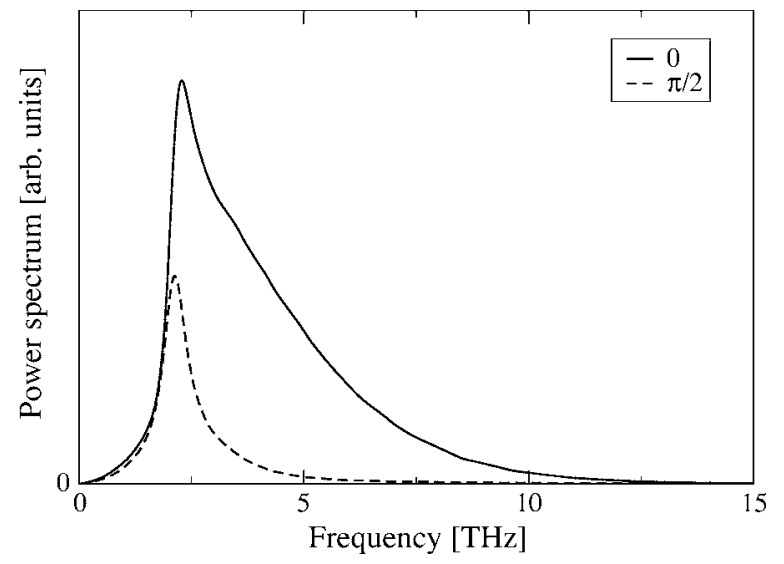

FIG. 4. Power spectrum of the far-field $\mathrm{THz}$ emission for two different optical phases, $\phi=0$ (solid line) and $\phi=\pi / 2$ (dashed line). Relaxation time $\tau=1$ ps. 


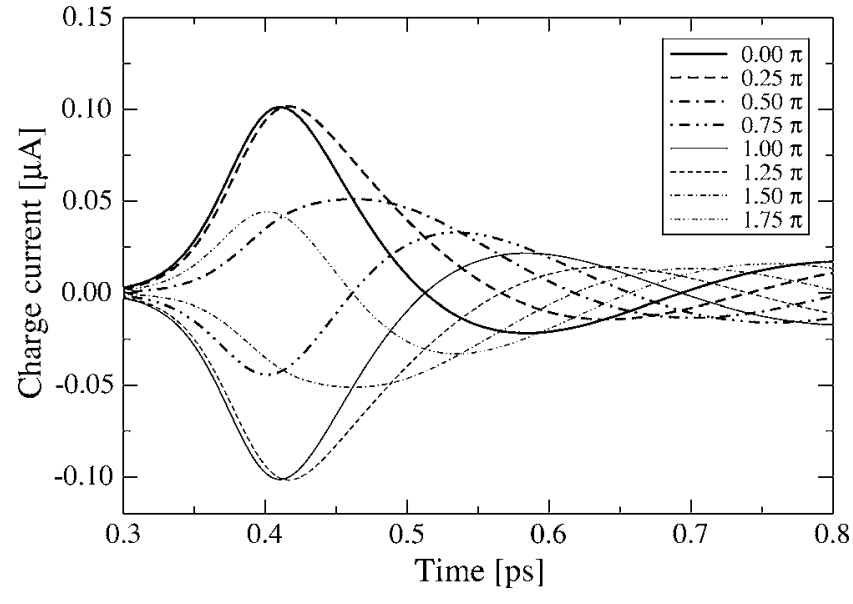

FIG. 5. Charge current along the V-shaped quantum wire for different optical phases $\phi$. Relaxation time $\tau=1$ ps.

one- and two-photon transitions. As discussed in Ref. 5, the same initial and final states are coupled by two coherent electromagnetic fields of frequency $2 \omega$ and $\omega$. The interferences between the two processes induce a charge current, whose amplitude can be controlled by the relative phase between the two pulses, the current is maximal for the phase $\phi=0$ and zero for the phase $\phi=\pi / 2$.

The oscillating charge current in Fig. 3 separates into two contributions. The amplitude of one part of the current can be controlled by the relative phase between the beams. In particular, it vanishes for $\phi=\pi / 2$. By contrast, the rest of the current cannot be switched off by tuning the phases of the pulses. The oscillations we are interested in appear in this second contribution. Figure 5 shows that the amplitude of the oscillating current (after the pulses vanished) is not affected by the optical phase $\phi$. Nevertheless, the phase of the oscillations can still be controlled.

In the terahertz emission, the oscillations of the current appear in both spectra as a sharp peak centered around 2 THz. In the case where an additional current is injected by tuning the phase to $\phi=0$, a broader contribution with higher frequency tail is superimposed on the peak.

We emphasize that in the present case, the optical frequencies are detuned with respect to the interband transition frequencies, as the QWR is excited slightly below the band gap. The injected charge current (and carrier densities) for $\phi=0$ would be much higher, if the subbands of the QWR were excited resonantly. However, in this paper, we focus on the excitonic contributions below the band gap.

In order to isolate and analyze the current oscillations, we concentrate now on the situation where no additional current is injected by interference between one- and two-photon transitions-i.e., $\phi=\pi / 2$. In this case, the peak in the power spectrum of the far-field terahertz emission is centered at $\sim 2.13 \mathrm{THz}$ ( or $\sim 8.8 \mathrm{meV}$ ).

\section{Analysis: Interference between $B_{1}$ and $A_{1}$ excitonic coherences}

The low-frequency oscillations in the polarization current are generated by interferences between $B_{1}$ and $A_{1}$ excitonic

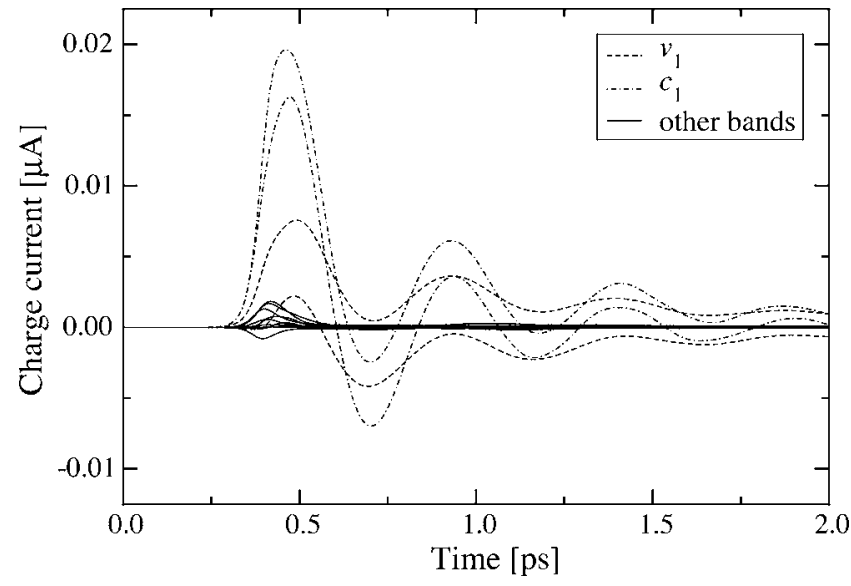

FIG. 6. Charge current in the different subbands. Relaxation time, $\tau=1$ ps. Optical phase, $\phi=\pi / 2$.

coherences stimulated, respectively, by the $\mathbf{A}_{1}$ and $\mathbf{A}_{2}$ electromagnetic fields. Indeed, the selection rules introduced in Sec. II B imply that the effective one- and two-photon interactions excite only excitons belonging to the irreps $B_{1}$ and $A_{1}$, respectively. The strong peak of the excitonic ground state in the one-photon absorption spectrum (Fig. 2) indicates that the $\mathbf{A}_{1}$-pulse primarily builds up the lowest $B_{1}$ excitonic coherence related to the subbands $c_{1}$ and $v_{1}$. On the other hand, the $\mathbf{A}_{2}$ pulse stimulates the $A_{1}$ excitonic resonances associated with the same subbands, that appear close to the band edge in the two-photon absorption spectrum (Fig. 2). The statement that this process involves mainly excitons related to the subbands $c_{1}$ and $v_{1}$ is confirmed by the small amount of current injectedin the other subbands. Indeed, Fig. 6 shows that the essential contribution to the charge current is given by the electrons in the subband pairs $c_{1}$ and $v_{1}$. The oscillatory motion of the electrons in the lowest conduction band $c_{1}$ is illustrated in Fig. 7, where we have plotted the carrier distribution-i.e., a diagonal element of the reduced density matrix - in reciprocal space, at different times during the evolution.

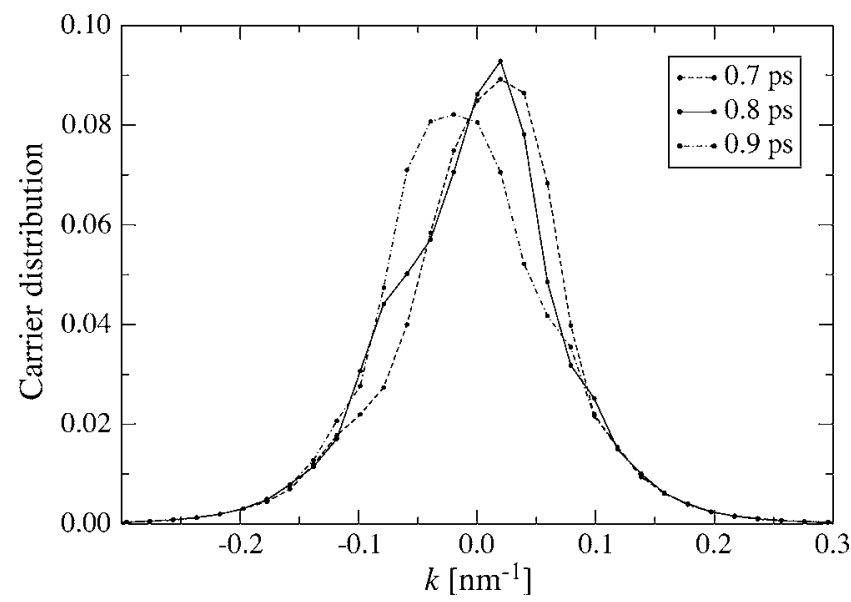

FIG. 7. Carrier distribution in the lowest pair of conduction subbands $c_{1}$ at different times $(0.7,0.8$, and $0.9 \mathrm{ps})$. The distribution is shown for the subband belonging to ${ }^{1} E_{1 / 2}$. The distribution in the conjugate subband belonging to ${ }^{2} E_{1 / 2}$ is similar. Optical phase, $\phi$ $=\pi / 2$. 
To get a better insight into the underlying physical process, let us illustrate this phenomenon by a simple two-band model. For this purpose, we consider an undoped semiconductor described by the occupation number $n_{k}=\rho_{c c, k}=1$ $-\rho_{v v, k}$ and the interband coherence $p_{k}=\rho_{c v, k}$. The semiconductor Bloch equations (SBE) that govern the dynamics can be written as

$$
\begin{gathered}
\frac{d}{d t} n_{k}=-\frac{2}{\hbar} \operatorname{Im}\left[\left(\mathbf{A} \boldsymbol{\Pi}_{k}+\sum_{q} V_{q} p_{k+q}\right) p_{k}^{*}\right], \\
\frac{d}{d t} p_{k}=-i \Omega_{k} p_{k}+\frac{i}{\hbar}\left(1-2 n_{k}\right)\left(\mathbf{A} \boldsymbol{\Pi}_{k}+\sum_{q} V_{q} p_{k+q}\right),
\end{gathered}
$$

where $\Omega_{k}$ is the renormalized interband frequency and $\boldsymbol{\Pi}_{k}$ $=\left(-e / m_{0}\right) \pi_{c v, k}$ is proportional to the interband momentum matrix element. After mixing the two equations, one is left with

$$
\frac{d}{d t}\left|p_{k}\right|^{2}=\left(1-2 n_{k}\right) \frac{d}{d t} n_{k} .
$$

Let us assume initial conditions that correspond to an unexcited system, $n_{k}(0)=p_{k}(0)=0$. One finds the relation

$$
n_{k}=\frac{1}{2}\left(1-\sqrt{1-4\left|p_{k}\right|^{2}}\right),
$$

valid for all times. For weak excitation, it reduces to $n_{k}$ $\simeq\left|p_{k}\right|^{2}$. Following the perturbative scheme described in Refs. 5 and 6, the dipole interaction in the SBE (5) can easily be replaced by two effective interaction terms, which are linear in the field of frequency $\omega_{1} \simeq \Omega_{k}$ and quadratic in the field of frequency $\omega_{2} \simeq \Omega_{k} / 2$, and which describe, respectively, resonant one- and two-photon transitions. Thus, the relation (7) remains valid for the interference process we want to discuss here.

Let us now assume that we excite simultaneously two excitonic resonances at frequencies $\Omega_{1}$ and $\Omega_{2}$, associated with the same two subbands. The corresponding coherence is given by

$$
p_{k}=\bar{p}_{1, k} e^{i \Omega_{1} t}+\bar{p}_{2, k} e^{i \Omega_{2} t},
$$

where, in the case of quasiresonant optical excitation, the functions $\bar{p}_{i, k}$ are slowly varying in time. The squared module of this coherence becomes

$$
\left|p_{k}\right|^{2}=\left|\bar{p}_{1, k}\right|^{2}+\left|\bar{p}_{2, k}\right|^{2}+2 \operatorname{Re}\left(\bar{p}_{1, k} \bar{p}_{2, k}^{*} e^{i\left(\Omega_{1}-\Omega_{2}\right) t}\right) .
$$

For weak excitation, this expression is almost equal to the carrier distribution $n_{k}$. As a consequence, the occupation number $n_{k}$ oscillates with frequency $\Omega_{1}-\Omega_{2}$. Now, if the coherences $\bar{p}_{1, k}$ and $\bar{p}_{2, k}$ are, respectively, even and odd as functions of the wave vector $k$, and of comparable magnitude, then the resulting carrier distribution is asymmetric in $k$ space, leading to a macroscopic charge current. The important point here, is that the excitonic frequencies do not depend on the wave vector $k$. Thus, all occupation numbers $n_{k}$ oscillate in phase. It follows that the related charge current oscillates with frequency $\Omega_{1}-\Omega_{2}$ given by the energy spacing between the involved excitonic resonances. This is what we observe in Figs. $3-7$. First, the lowest $B_{1}$ and $A_{1}$ excitonic coherences excited by the $\mathbf{A}_{1}$ and $\mathbf{A}_{2}$ pulses display, respectively, an (even) $S$-like and (odd) $P$-like shape in reciprocal space (not shown). Second, the central frequency of the peak in the $\mathrm{THz}$ power spectrum $(\sim 8.8 \mathrm{meV})$ corresponds approximatively to the energy spacing between the $B_{1}$ and $A_{1}$ excitons related to the bands $c_{1}$ and $v_{1}(\sim 8.6 \mathrm{meV})$ shown in Fig. 2. The small discrepancy is discussed in Sec. III D.

To summarize, the two pulses of frequency $2 \omega$ and $\omega$ build up two interband coherences, which interfere. This results in an asymmetric carrier distribution in the bands. The key point is that the beams excite two excitonic resonances of different energies. As a consequence, the corresponding coherences oscillate with different frequencies. This leads to an injected current that oscillates with frequency given by the energy spacing between the excitons.

\section{Discrepancy between the THz frequency and the $B_{1}-A_{1}$ energy spacing}

First, we note that the energies of the excitonic resonances shift with higher carrier density and that these shifts may not be fully physical since we have neglected screening and the Coulomb-hole correction. However there are also other compelling reasons for the main discrepancy at hand. At zero density, the lowest $B_{1}$ and $A_{1}$ excitons are separated by $\sim 9.7 \mathrm{meV}$. The absorption spectra shown in Fig. 2 are calculated for finite thermalized carrier distributions corresponding to the densities of energy and densities of carriers that are injected by the two laser pulses in the configuration for current generation, $\sim 2 \times 2 \times 10^{4}$ electrons $/ \mathrm{cm}$ at $\sim 75 \mathrm{~K}$ in the lowest pair of conduction bands and $\sim 2 \times 2$ $\times 10^{4}$ holes $/ \mathrm{cm}$ at $\sim 18 \mathrm{~K}$ in the highest pair of valence bands (the temperature is given by the average energy of the excited carriers). Nevertheless, these thermalized carrier distributions are not exactly equal to the optically injected distributions. The latter increase while the current is building up, and evolve during the subsequent oscillations and thermalization (see Fig. 7). This affects the excitonic energies.

Second, the $\mathbf{A}_{2}$ laser pulse excites also excitonic resonances at higher energy. Indeed, the two-photon absorption spectra in Fig. 2 shows that the absorption near the band gap and the one due to the lowest $A_{1}$ exciton are of comparable magnitude. These (bound or unbound) excitons also interfere with the $B_{1}$ exciton, and affect the current.

Finally, if one wishes to identify precisely the energy spacing between the excitons in the terahertz emission, then all contributions that come from the build-up and decay of the current should first be deconvoluted from the power spectrum.

\section{E. Coherent control of the oscillations}

Concerning the coherent control of the injected current, we note that the optical phases of the beams cannot be used to tune the amplitude of the oscillating current. This is related to the fact that, even in the stationary regime, there is no constant phase relation between the interfering coherences. However, one can modify the phase of the oscillations. By contrast, if the two beams would excite two resonances with identical energies, then the associated coher- 


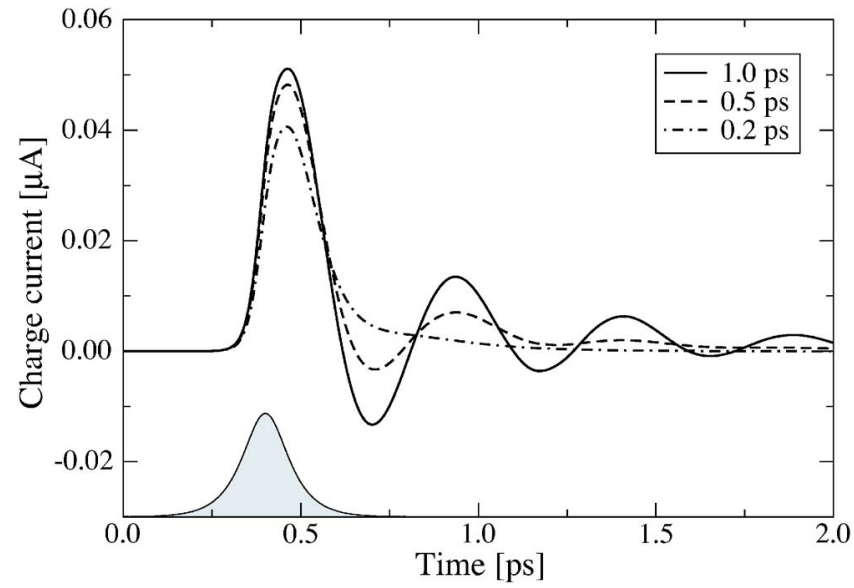

FIG. 8. (Color online) Charge current along the V-shaped quantum wire for different relaxation times $\tau(1 \mathrm{ps}, 0.5 \mathrm{ps}$, and $0.2 \mathrm{ps})$. Optical phase, $\phi=\pi / 2$. The shaded curve on the bottom of the figure shows the time dependence of the optical pulses in the QWR.

ences would oscillate in phase (with same frequency). In such a case, the current would not oscillate. However, the constant relative phase between the coherences could be shifted by the optical phases of the beams, which would allow to control the current injection rate for all times. This is the mechanism governing the coherent control of current injection $^{1-5}$ where the two optical fields resonantly excite two coherences between the same pair of conduction and valencebands, which oscillate both with the interband frequency.

\section{F. Dependence on the relaxation time}

In Fig. 8, we show the calculated time evolution of the optically injected charge current for different relaxation times $(1 \mathrm{ps}, 0.5 \mathrm{ps}$, and $0.2 \mathrm{ps})$ and $\phi=\pi / 2$. The power spectrum of the corresponding terahertz emission is displayed in Fig 9. One sees that the relaxation time must be sufficiently long, for the current oscillations to be observable. This implies low carrier densities, and thus weak excitation. On the other hand, the oscillations appear in the charge current, since the latter is directly related to carrier distribution in the bands. Therefore, from the experimental

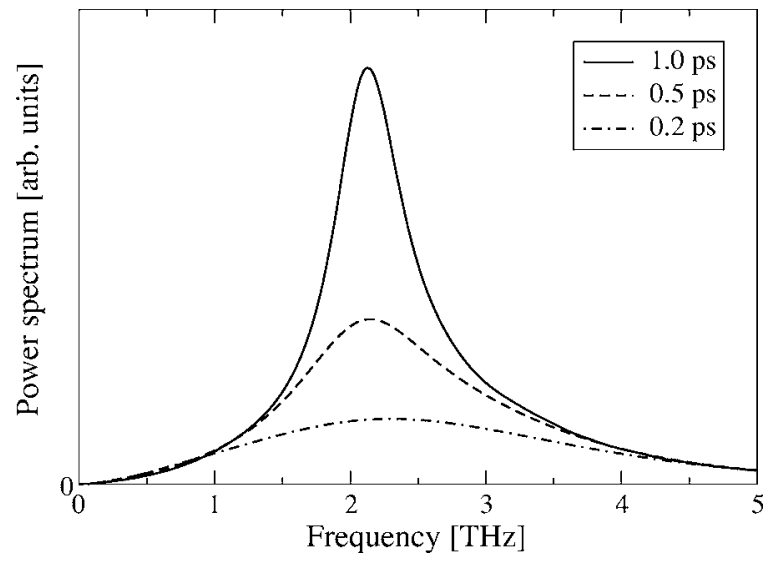

FIG. 9. Power spectrum of the far-field THz emission for different relaxation times $\tau(1 \mathrm{ps}, 0.5 \mathrm{ps}$, and $0.2 \mathrm{ps})$. Optical phase, $\phi$ $=\pi / 2$.

point of view, a stronger signal requires higher carrier densities, which accelerates the decay of the current. This tradeoff deserves further investigations.

\section{CONCLUSION}

Using the effective multiband Bloch equations ${ }^{5,6}$ including the Coulomb interaction within the Hartree-Fock approximation, we evidenced new oscillations in the charge current injected in a V-shaped AlGaAs/GaAs QWR, when excited slightly below the band gap by two phase-related femtosecond laser pulses of frequency $\omega$ and $2 \omega$. The origin of these oscillations could be attributed to the fact that the two optical beams excite independently the lowest $B_{1}$ and $A_{1}$ excitonic resonances. We have shown that the interferences between these excitonic coherences result in terahertz oscillations in the low-frequency polarization current, that subsist after the laser pulses vanished. For a long decoherence time $(1 \mathrm{ps})$, these oscillations appear as a distinct peak in the power spectrum of the far-field terahertz emission. By contrast to the current injected by resonant one- and two-photon interband transitions discussed in Ref. 5, this oscillating current cannot be switched off by tuning the relative phase between the two pulses.
${ }^{1}$ E. Dupont, P. B. Corkum, H. C. Liu, M. Buchanan, and Z. R. Wasilewski, Phys. Rev. Lett. 74, 3596 (1995).

${ }^{2}$ A. Haché, Y. Kostoulas, R. Atanasov, J. L. P. Hughes, J. E. Sipe, and H. M. van Driel, Phys. Rev. Lett. 78, 306 (1997).

${ }^{3}$ P. Král and J. E. Sipe, Phys. Rev. B 61, 5381 (2000).

${ }^{4}$ A. Najmaie, R. D. R. Bhat, and J. E. Sipe, Phys. Rev. B 68, 165348 (2003).

${ }^{5}$ D. H. Marti, M. A. Dupertuis, and B. Deveaud, Phys. Rev. B 69, 035335 (2004).

${ }^{6}$ D. H. Marti, M. A. Dupertuis, and B. Deveaud, Ann. Phys. (N.Y.) 316, 234 (2005).
${ }^{7}$ E. O. Gobel, K. Leo, T. C. Damen, J. Shah, S. Schmitt-Rink, W. Schafer, J. F. Muller, and K. Kohler, Phys. Rev. Lett. 64, 1801 (1990).

${ }^{8}$ K. Leo, T. C. Damen, J. Shah, E. O. Gobel, and K. Kohler, Appl. Phys. Lett. 57, 19 (1990).

${ }^{9}$ S. Schmitt-Rink et al., Phys. Rev. B 46, 10460 (1992).

${ }^{10}$ M. A. Dupertuis, E. Martinet, D. Y. Oberli, and E. Kapon, Europhys. Lett. 52, 420 (2000).

${ }^{11}$ F. Vouilloz, D. Y. Oberli, M. A. Dupertuis, A. Gustafsson, F. Reinhardt, and E. Kapon, Phys. Rev. B 57, 12378 (1998).

${ }^{12} \mathrm{G}$. Bastard, Wave Mechanics Applied to Semiconductor Hetero- 
structures (Les éditions de Physique, Les Ulis, France, 1990).

${ }^{13}$ C. Bosio, J. L. Staehli, M. Guzzi, G. Burri, and R. A. Logan, Phys. Rev. B 38, 3263 (1988).

${ }^{14}$ L. W. Molenkamp, R. Eppenga, G. W. 't Hooft, P. Dawson, C. T. Foxon, and K. J. Moore, Phys. Rev. B 38, 4314 (1988).

${ }^{15}$ S. L. Altmann and P. Herzig, Point-Group Theory Tables (Oxford University Press, New York, 1994).

${ }^{16} \mathrm{R}$. Zimmermann, in Spectroscopy and Dynamics of Collective
Excitations in Solids, edited by B. Di Bartolo and S. Kyrkos (Plenum, New York, 1997), pp. 123-145.

${ }^{17}$ M. A. Dupertuis, D. Y. Oberli, and E. Kapon, International Conference on Computational Nanoscience ICCN 2002 (Computational Publications, Puerto Rico, U.S.A., 2002).

${ }^{18}$ D. H. Marti, M. A. Dupertuis, and B. Deveaud, IEEE J. Quantum Electron. 41, 848 (2005). 